\title{
A METHOD OF 3D MEASUREMENT AND RECONSTRUCTION FOR CULTURAL RELICS IN MUSEUMS
}

\author{
Shunyi Zheng ${ }^{\mathrm{a}}$, Yang Zhou ${ }^{\mathrm{a}}$ *, Rongyong Huang ${ }^{\mathrm{a}}$, Langming Zhou ${ }^{\mathrm{a}}, \mathrm{Xuan}_{\mathrm{Xu}}^{\mathrm{a}}$, Chengyi Wang ${ }^{\mathrm{b}}$ \\ ${ }^{a}$ School of Remote Sensing and Information Engineering, Wuhan University, 129 Luoyu Road, Wuhan 430079, China- \\ (syzheng, zhouyang, hry)@whu.edu.cn, zlm_mj@126.com, xuxuan@263.net.cn \\ ${ }^{\mathrm{b}}$ Institute of Remote Sensing Applications, Chinese Academy of Sciences, P.O.BOX9718, Rd Datun, District Chaoyang, \\ Beijing, China- wangcycastle@163.com
}

\section{Commission V, WG V/2}

\begin{abstract}
KEY WORDS: Archaeology, Digitisation, Texture, Photo-realism, Structure-light, Cultural relics in museum, 3D measurement and
\end{abstract} reconstruction, Texture mapping

\begin{abstract}
:
Three-dimensional measurement and reconstruction during conservation and restoration of cultural relics have become an essential part of a modem museum regular work. Although many kinds of methods including laser scanning, computer vision and close-range photogrammetry have been put forward, but problems still exist, such as contradiction between cost and good result, time and fine effect. Aimed at these problems, this paper proposed a structure-light based method for 3D measurement and reconstruction of cultural relics in museums. Firstly, based on structure-light principle, digitalization hardware has been built and with its help, dense point cloud of cultural relics' surface can be easily acquired. To produce accurate 3D geometry model from point cloud data, multi processing algorithms have been developed and corresponding software has been implemented whose functions include blunder detection and removal, point cloud alignment and merge, 3D mesh construction and simplification. Finally, high-resolution images are captured and the alignment of these images and 3D geometry model is conducted and realistic, accurate 3D model is constructed. Based on such method, a complete system including hardware and software are built. Multi-kinds of cultural relics have been used to test this method and results prove its own feature such as high efficiency, high accuracy, easy operation and so on.
\end{abstract}

\section{INTRODUCTION}

Building 3D models with high accuracy and realistic texture is a very significant part in the field of digital museum, heritage conservation, relics appreciation and exhibit, etc. Although many units of heritage sector and associated professional companies have made a great deal of effort to resolve the related issues, it is still a long way to fast, realistically and accurately measure and reconstruct the relics in museums. In which the most concerned problem is the contradiction between cost and good result, time and fine effect.

At present, general-used methods in 3D measurement include close-range photogrammetry, computer vision and laser scanning etc. Photogrammetry, a branch of the surveying and mapping, has played an important role in Conservation and Archaeology in recent years, which is mainly used for archaeological measurement of field site, stone carving and caves etc. But because of its low-quality point cloud, the applications in high-quality modelling cultural relics are few. Besides, the complicated process such as calibration and aerotriangulation, is another cause that photogrammetry does not fit for 3D reconstruction of cultural relics in museums. Compared to photogrammetry, laser scanning can quickly acquire dense point cloud of object surface. However, it has many deficiencies too. When the object is dark, the quality and reliability of scan shrinks, which is due to the reduction of reflection ratio. To texture mapping, it is low efficient since it needs third-party software, which involves much manual work and is very time consuming. And when the surface varies significantly, the alignment for point clouds often needs artificial targets, which is not allowed for most cultural relics. In a word, because of these shortcomings, there is still no method to settle all the relevant problems completely for 3D measurement and reconstruction of cultural relics in museums.

Combined with our photogrammetric background and experiences in cooperation with the heritage department, this paper proposed a novel method for 3D reconstruction of the cultural relics in museums. Experiments proved that our approach has many advantages, and we hope it could contribute to the progress of $3 \mathrm{D}$ measurement and reconstruction of cultural relics.

\section{POINT CLOUD ACQUISITION}

Accurately extracting the object's geometry information is the basis of 3D measurement and reconstruction of relics. And to make the model reflecting the object's shape precisely and fine, it is primary to acquire the dense 3D point set of the surface, which is also called 3D point cloud(Kan 2011).

There are various relics with different materials, colors and shapes. When the relics are lack of texture, it is hard to conduct 3D measurement on their surface through traditional photogrammetric technique. In contrast, the structure-light based method is superior in this respect via making use of assistant light to overcome the limit of texture lack. Meanwhile the point cloud acquired by structure-light system is more reliable, precise and dense. Since the process of aerotriangulation is omitted, its operation is easier. And

\footnotetext{
* Corresponding author.
} 
compared to laser scanning, the cost of structure-light is lower but it even can deal with the dark objects in weak reflections.

The general process of structure-light system includes: calibration of system, design of projection raster, calculation of corresponding stripes, generation of 3D point cloud, and alignment of different scans etc. Details of the work flow are as follows: the raster generator (projector) casts the designed stripes, at the same time one or two digital camera photograph the projected surface; then the computer decodes the captured images and calculates the corresponding stripes between the image and the raster (or the other image); at last 3D coordinates can be computed according to the parameters of camera and the relative parameters between cameras, thus the dense point cloud is produced. The structure-light photogrammetry system is flexible, in which the operations can be chosen according to specific requirements but not confined to some particular way.

The photogrammetry scanning system shown in Figure1 is designed by Institute of Digital Photogrammetry and Computer Vision, Wuhan University. The object is put on the rotation stage. After clicking the "Start" button on the hardware's controlling software, the system begins scanning. It takes about 10 seconds for a scan, and the stage will rotate an angle (the number can be pre established) after that till a whole round finished. The entire process is automatic, and its result is automatically aligned dense point cloud of the object surface (as illustrated in Figure2). For the top \& bottom areas of relics, or where invisible, additional scans from specific locations are usually necessary supplement in order to ensure the integrality of 3D point data.

\section{THREE-DIMENSIONAL GEOMETRY MODEL}

Dense point cloud is the basis of 3D measurement and reconstruction, but not the end product. In order to improve the exhibition effect of 3D shape, it is needed to further process the point data into 3D geometry model, including gross error elimination, alignment, merge, mesh reconstruction etc.

After analysis of the obtained point data, we found that the gross errors are usually isolated points, while the correct points are of good continuity and connectivity. Based on this thesis, we first detect the isolated points and small point group, and then eliminate them as gross errors for improving the quality of point data. Owing to the help of rotation stage, most point clouds have been aligned during acquisition, which means that the coordinates of different scans are unified. And for the additional scans, they can be aligned by manual aided method: first select 3 or more pairs of corresponding points, and then execute ICP algorithm for exact alignment(Besl and McKay 1992; Yang and Medioni 1992). However, ICP algorithm will degenerate in some special situations(Gelfand, Ikemoto et al. 2003). Considering of this shortage, we can use the imagebased alignment method, of which the images is captured by the camera of structure-light system during scanning and these the synchronized images will used later in texture mapping too. This is where the structure-light measurement system is superior to the laser scanning. Then merge is carried out after alignment by combining the multiple aligned point clouds (multi-layer) into one layer, for ensuring the uniformity of point data. The later process is $3 \mathrm{D}$ mesh reconstruction, which is to establish the topologies between the 3D surface points. After this step, the 3D shapes are no longer as discrete point set, but as models of triangulated mesh (as shown in Figure3).

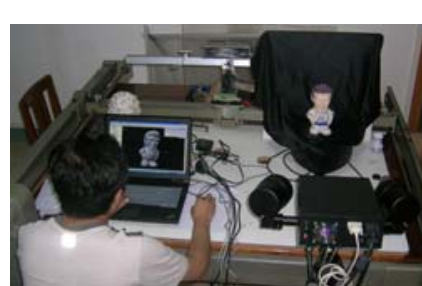

Figure 1. Scanning System
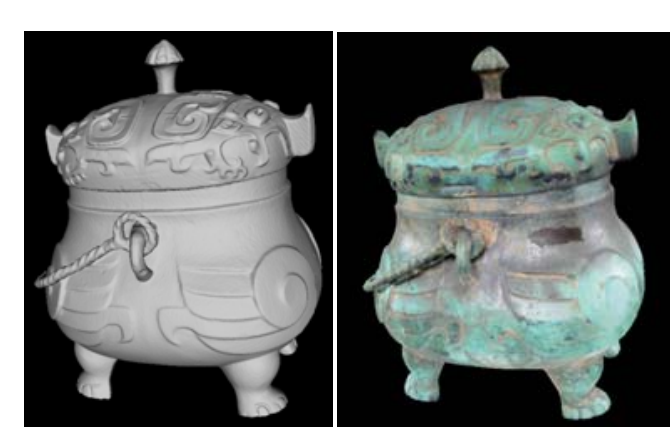

Figure3. 3D Geometry Model Figure 4. 3D Model with Texture

\section{TEXTURE MAPPING}

In order to realistically show the materials and colors of relics, it is needed to map the texture images on the geometry model (as rendered in Figure4). Since the structure-light system is still an image-based measurement method, the texture images have been collected during the course of scanning. However, those images captured by the industrial camera are not proper for high-quality and realistic texture mapping, because of low resolution and distorted colors. It's better to use the professional camera of high-resolution to acquire texture images in specialized photography environment. Then the main issue is focused on how to map these images of high-resolution and vivid colors onto the geometry models. It consists of two parts: (1). How to restore the orientation parameters of the highresolution photos to the object model, which is so called registration; (2). How to generate seamless texture from multiview images.

Some commercial software such as PolyWorks and MAYA has the texturing function, whose registration is making use of $\mathrm{N}$ pairs of corresponding points between images and geometry models to solve the orientation parameters. But those corresponding points are usually selected by hand, which is heavy workload and poor accuracy. The linear feature based methods(Ayman, Mwafag et al. 2005) are accurate, but mainly used in the field of building reconstruction and urban surveying, where the linear features are sufficient and distinct. Another multi-view based accurate method first conducts photogrammetric processing on the high-resolution images, and then perform registration between the photogrammetric and the scanning point cloud to restore the orientation parameters(Fei 2006). The course of this approach includes the entire photogrammetric process, which is too complicated, and moreover it can't be used to register one single image. By taking full advantage of structure-light scanning system, we proposed a novel registration method by regarding the synchronized images as references to solve the orientation parameters of high-resolution images(ZHENG Shunyi 2012). First running image matching between the synchronized and high-resolution images, and then casting a ray from the 
synchronized image to the mesh to calculate the object space coordinates of the matching points. At last according to the corresponding points between objects and images, the orientation parameters will be computed by the RANSAC based robust resection method. Figure5 illustrates a sketch map of our registration method. It's actually a process of using image matching and ray cast to replace the manual work of selecting corresponding points, therefore it is highly automated and efficient, and as a result of the improvement of the exactness of the corresponding points, the accuracy and reliability raises too.

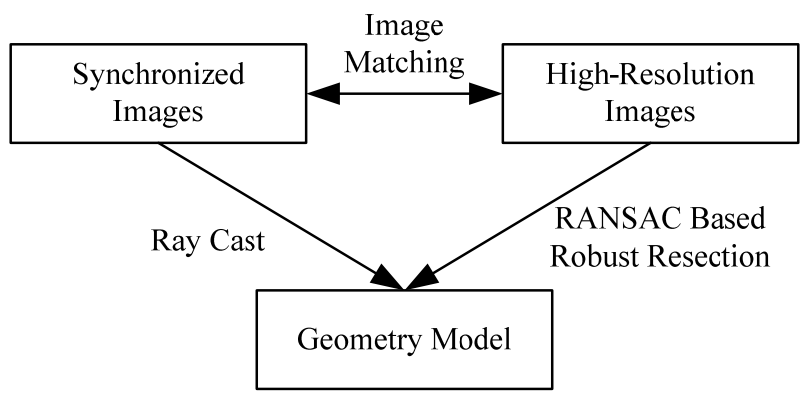

Figure 5. The Sketch Map of Automatic Registration for HighResolution Images

The texture seams can be attributed to two inaccuracies: the geometric and the photometric. The former includes geometry error of point cloud, the registration error, and the perspective distortion etc.; the photometric inaccuracies are due to the variations of light condition, sensitization of camera and the surface reflecting etc. To remove the visible seams, first using the Markov Random Field based method to achieve geometric align(Lempitsky and Ivanov 2007), and then taking the multiband blending or the Poisson Image Editing process to achieve the photometric align(Allene, Pons et al. 2008; Gal, Wexler et al. 2010), and thus realizing the truly seamless texture mapping. Figure6 is a set of result comparison between the traditional and seamless texture mapping. The display effect is obviously improved.

\section{EXPERIMENTS AND ANALYSIS}

For validating our method, PHOTOSCANSHOP - a complete system including hardware and software, built by Institute of Digital Photogrammetry and Computer Vision, Wuhan University, China and Wuhan Ruizhi Vision Measurement Technology Co. Ltd, China-is used to carry out experiments in $3 \mathrm{D}$ measurement and reconstruction of cultural relics in museums. Multi kinds of relics have been reconstructed through this system, which proved that our method has the specialties of high efficiency, high accuracy and high reality.

Table 1. The Calibration Results in High-accuracy Plane Calibration Board

\begin{tabular}{cccc}
\hline $\begin{array}{c}\text { Scanning } \\
\text { Distance }\end{array}$ & $\begin{array}{c}\text { Global } \\
\text { Planeness }\end{array}$ & $\begin{array}{c}\text { Planeness After Gross } \\
\text { Error Elimination }\end{array}$ & $\begin{array}{c}\text { Local } \\
\text { Planeness }\end{array}$ \\
\hline $42 \mathrm{~cm}$ & $0.059 \mathrm{~mm}$ & $0.057 \mathrm{~mm}$ & $0.035 \mathrm{~mm}$ \\
$51 \mathrm{~cm}$ & $0.069 \mathrm{~mm}$ & $0.060 \mathrm{~mm}$ & $0.045 \mathrm{~mm}$ \\
$60 \mathrm{~cm}$ & $0.088 \mathrm{~mm}$ & $0.069 \mathrm{~mm}$ & $0.058 \mathrm{~mm}$ \\
\hline
\end{tabular}

Table 1 is the calibration results for validating the accuracy of PHOTOSCANSHOP by using the high-accuracy plane calibration board, from which we could see that the relative accuracy of the system has reached the level of ten-thousandth, and hence the precision of reconstruction is guaranteed.

As an instance, Figure7 shows the 3D reconstruction process of a doll, which was taken 8 scans of a round on the rotation stage (about one and half minutes). The 8 collected synchronized images (with resolution of $1280 * 1024$ ) are shown at the upper half of the Figure 7(a), and Figure 7(c) shows the obtained point clouds which have been aligned automatically during scanning. After simple editing on points data, it could go along the operations of gross error elimination, points merge, normal evaluation, and mesh reconstruction etc step by step. These operations totally took about 15 20 minutes, and the resulting $3 \mathrm{D}$ geometry model is the triangulated irregular network as rendered in Figure 7(d). After that all the synchronized images could be mapped onto the model. And the example result is shown in Figure 7(e), which was taken about half minute for texture computing. From Figure 7(e), we could see that the colors of the synchronized images were seriously distorted and the brightness was dark. The lower half of Figure 7(a) lays out the photos shot by SLR cameras (with resolution of 4288*2848), which can be quickly registered one by one through our method. Figure 7(b) shows the image matching result of the front \& back face of the doll in the registration process of our method. By using the GPU-based SIFT matching, our registration method is very efficient, which takes less than 10secs for one high-resolution image. After finishing sufficient registration, the high quality and high realistic seamless texture model could be generated in 1 minute as the result of the doll rendered in Figure 7(f). In summary, the whole process of reconstruction for the doll was less than half-hour, or even an hour with calculating the time of photographing high-resolution images.

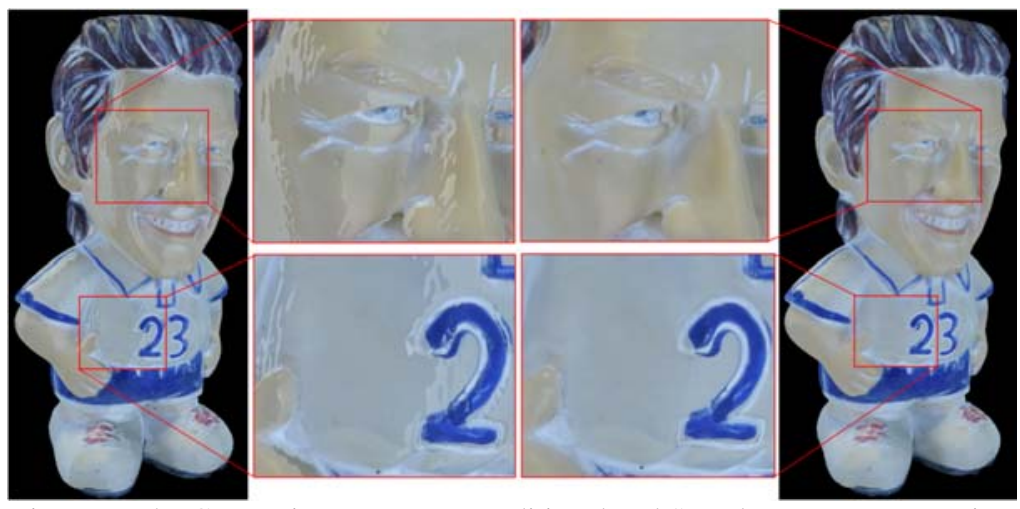

Figure 6. The Comparison Between Traditional and Seamless Texture Mapping 


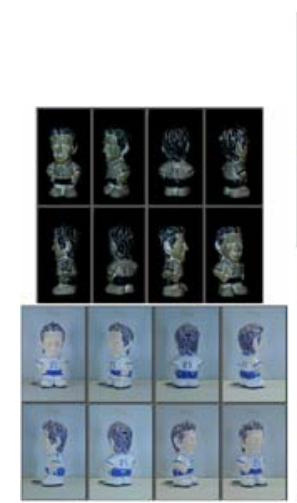

(a)

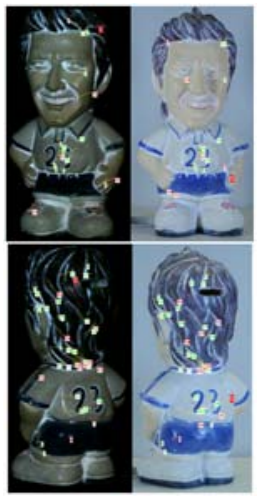

(b)

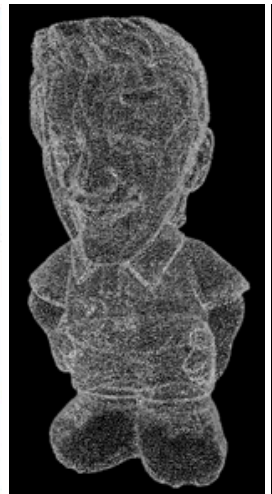

(c)

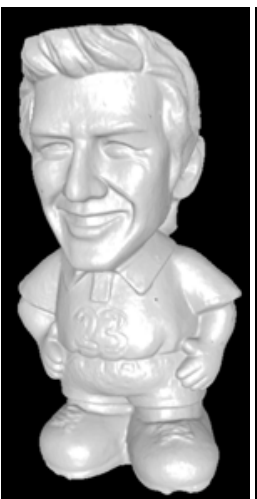

(d)

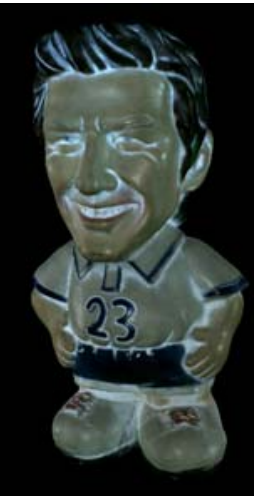

(e)

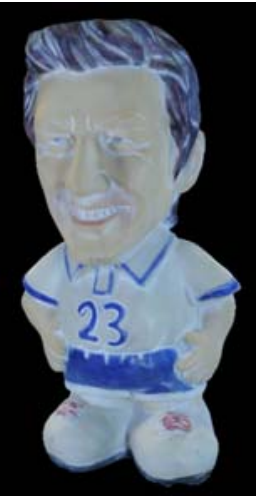

(f)

Figure 7. The 3D Reconstruction Process of a Doll

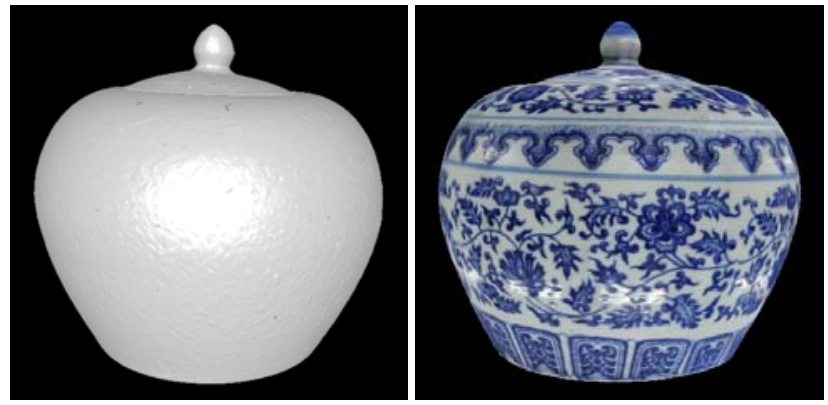

(a) A Bule and White Porcelain
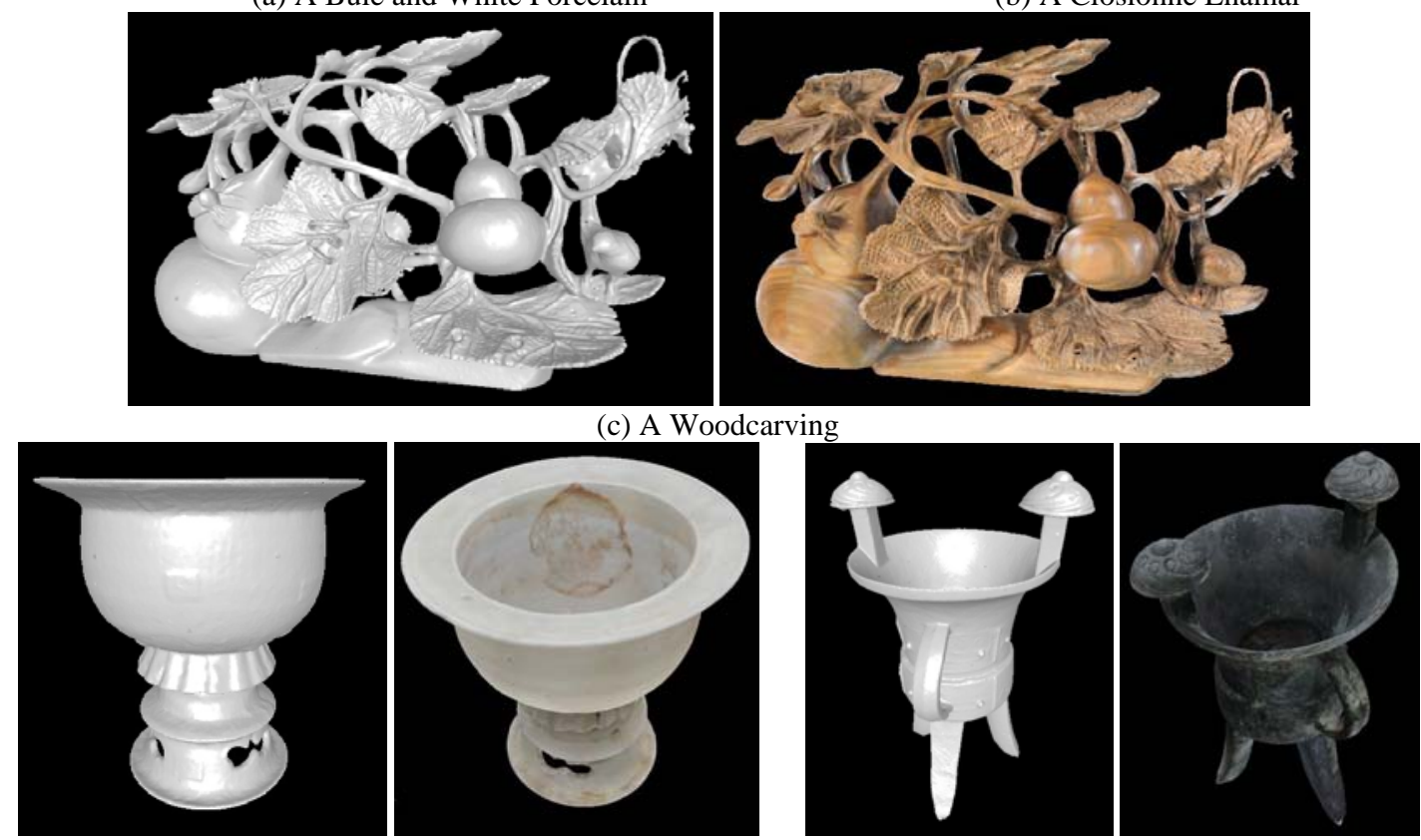

(d) The Cup-Furnace in Xiaogan Museum, Hubei, China (e) The Bronze-Jia in Xiaogan Museum, Hubei, China

Figure 8. The 3D Reconstruction Results of Some art and craft goods and cultural relics in museums

Figure 8 exhibits the other experimenting results, from ordinary art and craft goods to precious cultural relics in museums, which are all accurate, vivid and true like, proved the powerful ability of 3D reconstruction of our method.

\section{CONCLUSIONS}

Aimed at the problems of low efficiency (especially in texture mapping), high cost, and poor effect etc. of the current methods, this paper proposed a novel approach based on the structurelight scanning system to conduct 3D measurement and reconstruction of cultural relics in museums. The workflow includes point cloud acquisition, gross error elimination, data alignment and merge, 3D mesh reconstruction, and texture mapping etc. Its end product is the high accurate and vivid 3D geometry model with realistic textures. We developed an intact set of measurement system. Plenty of tests on this system proved the validity and superiority of our approach. 
Acknowledgements This work is supported by the Chinese National Natural Science Foundation Program (No. 41071293).

\section{REFERENCES}

Allene, C., J. P. Pons, et al. (2008). Seamless image-based texture atlases using multi-band blending. Pattern Recognition, 2008. ICPR 2008. 19th International Conference on.

Ayman, H., G. Mwafag, et al. (2005). "Photogrammetric and lidar data registration using linear features." Photogrammetric engineering and remote sensing 71(6): 9.

Besl, P. J. and N. D. McKay (1992). "A Method for Registration of 3-D Shapes." IEEE Trans. Pattern Anal. Mach. Intell. 14(2): 239-256.

Fei, D. (2006). Research on LIDAR and Digital Images Registration and Objects Extraction. School of Remote Sensing and Information Engineering. Wuhan, Wuhan University. PhD.

Gal, R., Y. Wexler, et al. (2010). "Seamless Montage for Texturing Models." Computer Graphics Forum 29(2): 479-486.

Gelfand, N., L. Ikemoto, et al. (2003). Geometrically stable sampling for the ICP algorithm. 3-D Digital Imaging and Modeling, 2003. 3DIM 2003. Proceedings. Fourth International Conference on.

Kan, D. (2011). "Research on the application of digital modeling technology in the conservation of cultural relics in museums." Sciences of Conservation and Archaeology 23(1): 62 66.

Lempitsky, V. and D. Ivanov (2007). Seamless Mosaicing of Image-Based Texture Maps. Computer Vision and Pattern Recognition, 2007. CVPR '07. IEEE Conference on.

Yang, C. and G. Medioni (1992). "Object modelling by registration of multiple range images." Image and Vision Computing 10(3): 145-155.

ZHENG Shunyi, Z. Y. (2012). "High Quality Texture Reconstruction for Small Objects Based on Structure-light Scanning System with Digital Camera." Geomatics and Information Science of Wuhan University(In Press). 